\title{
Modified PFA with the Azimuth Resampling in Post Fourier Transformed Data Domain
}

\author{
Liang Lu, Pan Li \\ Department Information Engineering \\ Lanzhou Vocational Technical College \\ No. 37 Anning Liusha Road, Lanzhou, 730070, China \\ Phone: 15393126628 \\ E-mail: LL_MAIL04@126.com, 809191387@,QQ.COM
}

\author{
Pan Li \\ College of Physics and Electronic Engineering \\ Northwest Normal University \\ No. 967 Anning East Road, Lanzhou, 730070, China \\ E-mail: $\underline{632459924 @ \text { QQ.COM }}$
}

\begin{abstract}
The polar format algorithm (PFA) is the most popular way for spotlight synthetic aperture radar (SAR) image formation and is still attractive nowadays. Conventionally, the algorithm is carried out sequentially as a range resampling, an azimuth resampling, a Fourier transform (FT) in azimuth, and an FT in range. However, this procedure usually leads to zerospadding in the azimuth resampled data domain, and consequently degrades final image quality. In this paper, a modified PFA with the azimuth resampling in post azimuth Fourier transformed data domain, as opposed to traditionally done in pre-transformed domain is studied. The modification relies on the frequency scaling property of the FT. To ensure the efficiency of the PFA, we adopt the fast FT (FFT) based scaling scheme for the azimuth resampling. Besides, a processing flow is proposed for real time application. The proposed PFA performs well and efficiently in the imaging of simulated and real spotlight SAR data.
\end{abstract}

Keywords-spotlight synthetic aperture radar (SAR); polar format algorithm (PFA); Fourier transform (FT)

\section{INTRODUCTION}

Synthetic aperture radar (SAR) spotlight mode [1], [2] allows the generation of microwave images with high geometric resolution by steering its antenna beam to continuously illuminate the same scene on the ground (spot) to increase the synthetic aperture time for high resolution in azimuth, and the high resolution in range can be achieved by transmitting signal with large bandwidth.

In spotlight SAR image formation, the polar format algorithm (PFA) [1] is a simple and efficient method for generating high resolution imagery over a reasonably imaging area. Of a PFA, the acquired spotlight SAR data are resampled from a polar format into a rectangle (Cartesian) grid. After a 2dimentional (2-D) FT in azimuth and range respectively, a focus SAR image is obtained. A tandem interpolation strategy is traditionally used along range and azimuth for the resampling, however, this can be time consuming [3]. Therefore, a method to facilitate the resampling while preserving the performance is valuable. For example, Doerry [4] designed the SAR system by varying transmitting parameters,

This study is supported by Central Financial "support for higher vocational schools to enhance the development capacity of the professional services industry" project. such as the center frequency and chirp rate, on pulse by pulse basis for the range resampling and then performed the chirp- $Z$ transform (CZT) for the azimuth resampling. Both of the interpolations in range and azimuth are avoided for improving efficiency. Zhu et al. [5] adopted the scaling scheme for the resampling in range and azimuth, and the scheme could be achieved by fast FT (FFT) implementation.

Conventionally, as for the processing flow, two tandem resampling in range and azimuth are performed first, and then two sequential FT operations in azimuth and range are used to focus the SAR image. After the azimuth resampling, zeropadding for areas without resampled data in the 2-D Cartesian data domain of azimuth and range wavenumbers is needed before the azimuth FT. Consequently, sidelobes of the azimuth response after the FT can increase due to the padding.

To avoid the padding, a modified PFA is studied by performing the azimuth resampling after the azimuth FT. Thus, the entire useful data samples in the full-filled data domain along azimuth can be utilized for improving the focusing quality. The modification is guaranteed in theory with the frequency scaling property of the FT. Also, the parameter variation and azimuth scaling scheme are adopted to make the PFA efficient. Finally, simulation in spotlight mode is carried out to validate the proposal, and comparisons are listed in testifying its superiority. Real spotlight SAR data also prove the modified PFA.

\section{CONVEntional PFA}

In general, SAR transmits microwave signals in pulse repetition towards imaging scene on the ground, and collects echoes in polar coordinates. Suppose the transmitting signal in form of linear frequency modulation (LFM) is denoted as $s_{\mathrm{T}}(\cdot)$, the demodulated echo spectra of a point scatterer at $\left(x, y+Y_{c}\right)$ in the slant plane can be written in terms of range frequency $\Delta f_{r}$ as

$$
\begin{aligned}
s\left(\Delta f_{r}, n\right)= & \sigma(x, y) s_{\mathrm{T}}\left(\Delta f_{r}\right) . \\
& \exp \left[-\mathrm{j} \frac{4 \pi}{\mathrm{c}}\left(f_{c n}+\Delta f_{r}\right) R_{n}\right] \exp [\mathrm{j} \phi(n)]
\end{aligned}
$$


where $\Delta f_{r}$ corresponds to the Fourier transformed variable of fast time, $n$ is the pulse number, $\sigma(x, y)$ is the backward scattering amplitude of the point scatterer, $f_{c n}$ is the center frequency at pulse $n, c$ is the speed of light. $R_{n}$ denotes range history between the scatterer and the SAR sensor in ideal trajectory, $\phi(n)$ represents phase error related to the trajectory deviation. Thanks to the inertial navigation instrument [6] that can be used to compensate for the deviation in some extent, we ignore the range cell migration caused by the deviation, and only take the phase error into consideration in (1). After range matched filtering by multiplying conjugate of $s_{\mathrm{T}}\left(\Delta f_{r}\right)$, the digitalized echo spectra in wavenumber domain can be expressed as

$$
s(m, n)=\sigma(x, y) \exp \left[-\mathrm{j}\left(k_{c n}+m \Delta k_{n}\right) R_{n}\right] \exp [\mathrm{j} \phi(n)]
$$

In (2), $k_{c n}=\frac{4 \pi}{\mathrm{c}} f_{c n}$ is the center wavenumber for pulse $n$, $\Delta k_{n}=\frac{4 \pi}{\mathrm{c}} \Delta f_{r}$ is the wavenumber interval of pulse $n$. Because of the transmitting signal form in LFM, $\Delta f_{r}$ can be formulated as $\Delta f_{r}=\gamma_{n} \Delta \mathrm{T}_{\mathrm{p}}$ with $\gamma_{n}$ as the chirp rate of pulse $n$, and $\Delta \mathrm{T}_{\mathrm{p}}$ is the sample interval of pulse generation time and $m$ is the frequency sample index in range.

By setting $d$ to be the spatial interval of pulse repetition, and $(n d, 0)$ as the position of SAR sensor in ideal trajectory, the range history $R_{n}$ is approximated with first order Taylor expansion as

$$
\begin{aligned}
R_{n} & =\sqrt{(x-n d)^{2}+\left(y+\mathrm{Y}_{\mathrm{c}}\right)^{2}} \\
& \approx \sqrt{(n d)^{2}+\mathrm{Y}_{\mathrm{c}}^{2}}-\frac{n d}{\sqrt{(n d)^{2}+\mathrm{Y}_{\mathrm{c}}^{2}}} x+\frac{\mathrm{Y}_{\mathrm{c}}}{\sqrt{(n d)^{2}+\mathrm{Y}_{\mathrm{c}}^{2}}} y
\end{aligned}
$$

According to principle of the PFA, the approximation in (3) relies on the assumption that the planar wavefront propagates [7], which restricts spatial extent of a SAR image formed by PFA. To accommodate for image formation in large size, the space-variant filtering method proposed in [7] can be used to correct the wavefront curvature effect.

In the traditional PFA, the azimuth de-chirping technique [3] is utilized first, and then two tandem 1-D operations of resampling in the range and azimuth are applied respectively as

$$
\left\{\begin{array}{l}
\left(k_{c n}+m \Delta k_{n}\right) \frac{\mathrm{Y}_{c}}{\sqrt{(n d)^{2}+\mathrm{Y}_{\mathrm{c}}^{2}}}=k_{c 0}+m^{\prime} \Delta k_{0} \\
\left(k_{c 0}+m^{\prime} \Delta k_{0}\right) \frac{d}{\mathrm{Y}_{\mathrm{c}}} n=k_{c 0} \frac{d}{\mathrm{Y}_{\mathrm{c}}} n^{\prime}
\end{array}\right.
$$

$k_{c 0}=\frac{4 \pi}{c} f_{c 0}$ is the nominal center wavenumber with nominal center frequency $f_{c 0} \cdot \Delta k_{0}=\frac{4 \pi}{\mathrm{c}} \gamma_{0} \Delta \mathrm{T}_{\mathrm{p}}$ is the nominal wavenumber interval, and $\gamma_{0}$ is the nominal chirp rate. After resample the data from the polar format coordinates in $(m, n)$ to the Cartesian grid in $\left(m^{\prime}, n^{\prime}\right),(2)$ is given as

$$
\begin{aligned}
s\left(m^{\prime}, n^{\prime}\right)= & \sigma(x, y) \exp \left[\mathrm{j}\left(k_{c 0} \frac{d}{\mathrm{Y}_{c}} n^{\prime}\right) x\right] . \\
& \exp \left[-\mathrm{j}\left(k_{c 0}+m^{\prime} \Delta k_{0}\right) y\right] \exp \left[\mathrm{j} \phi\left(n^{\prime}\right)\right]
\end{aligned}
$$

Using the famous phase gradient autofocus (PGA) [8] method, $\phi\left(n^{\prime}\right)$ can be removed from the echo data, and the scatterer can be focused in range $y$ and azimuth $x$ by range and azimuth FFT, respectively.

\section{MODIFIED PFA WITH THE AZIMUTH RESAMPLING IN POST FOURIER TRANSFORMED DATA DOMAIN}

However, due to the coordinate transformation from polar format into Cartesian grid by the PFA resampling as (4), there must be zero-padding in azimuth. Consequently, when we use the azimuth FFT to accomplish the SAR image formation, the focusing quality will be degraded, such as increasing sidelobes. What is more, in implementation of the resampling by the interpolation operation, computational efficiency can be low, and it is impractical for real time SAR processing. In this paper, a modified PFA with the azimuth resampling in post azimuth FT data domain is proposed. Besides, in order to enhance the efficiency of the proposal, the parameter variation method in [4] is adopted for range resampling, and the FFT based scaling scheme [5] is utilized for azimuth resampling after the azimuth FFT.

According to the range resampling function of the first equation in (4), it can be separated as

$$
f_{c n} \frac{\mathrm{Y}_{\mathrm{c}}}{\sqrt{(n d)^{2}+\mathrm{Y}_{\mathrm{c}}^{2}}}=f_{c 0} \quad \text { and } \quad \gamma_{c n} \frac{\mathrm{Y}_{\mathrm{c}}}{\sqrt{(n d)^{2}+\mathrm{Y}_{\mathrm{c}}^{2}}}=\gamma_{c 0}
$$

The center frequency $f_{c n}$ can vary as the first equation shown in (6) on pulse by pulse basis. Similarly, the transmitting chirp rate can vary as the second equation shown in (6). By transmitting the signal with the parameter variation as (6) from pulse to pulse, range resampling can be accomplished avoiding the time consuming interpolation. At present, the received echo signal can be re-expressed as

$$
s(m, n)=\sigma(x, y) \exp \left(-\mathrm{j} m \Delta k_{0} y\right) \exp \left[\mathrm{j}\left(k_{c 0}+m \Delta k_{0}\right) n \frac{d}{\mathrm{Y}_{\mathrm{c}}} x\right]
$$

where the constant phase term corresponding to $k_{c 0} y=\frac{4 \pi}{\lambda} y$ is omitted, and $\lambda$ is the radar wavelength. Apparently, the phase 
term in the first exponent relates to the position of the scatterer in range $y$, where the samples are uniformly spaced at a constant interval of $\Delta k_{0}$. Thus, IFFT operation in range can be used to focus the scatterer in range. The phase term in the second exponent denotes the interval of azimuth samples. However, the interval varies with the range sample index $m$. The azimuth resampling is usually applied to resample the azimuth data in uniform interval as

$$
\left(k_{c 0}+m \Delta k_{0}\right) \frac{d}{R_{c}} n=k_{c 0} \frac{d}{R_{c}} n^{\prime} \Leftrightarrow \frac{k_{c 0}+m \Delta k_{0}}{k_{c 0}} n=n^{\prime}
$$

Furthermore, for any fixed range index as $m=m_{0}$, the interval of azimuth samples can be given as a constant value of $\frac{k_{c 0}+m_{0} \Delta k_{0}}{k_{c 0}}$. Thus, the azimuth samples in each range wavenumber index are also uniformly spaced. We call this property as linear resampling, which makes the resampling of the azimuth data after the azimuth FFT feasible. To prove the significance of the linear resampling, we restate the frequency scaling property of the Fourier transform,

$$
\text { if } \operatorname{FT}[g(n)]=G(h) \text { then } \operatorname{FT}[g(a n)]=G\left(\frac{h}{a}\right)
$$

FT [.] represents operation of Fourier transform. $a$ is a scaling coefficient. $g(\cdot)$ and $G(\cdot)$ are vectors of the same length. From (9), one can resample the original data $g(n)$ to get $g(a n)$ and perform an FT to obtain $G\left(\frac{h}{a}\right)$. Alternatively, the resampling can be applied to $G(h)$ to achieve $G\left(\frac{h}{a}\right)$. Therefore, before the azimuth resampling, we carry out an FFT to (7) and get

$$
\begin{array}{r}
s(m, h)=\operatorname{FFT}_{n}[s(m, n)]=\sigma(x, y) \exp \left(-\mathrm{j} m \Delta k_{0} y\right) . \\
\sum_{n} \exp \left\{\mathrm{j}\left(\left(\left(k_{c 0}+m \Delta k_{0}\right) \frac{d}{\mathrm{Y}_{c}} x\right)-\frac{2 \pi}{N} h\right] n\right\}
\end{array}
$$

$\mathrm{FFT}_{n}$ is the FFT operation in azimuth $n$, and $h$ stands for image pixel in azimuth. The summation term in the last equation of (10) has a sinc form and its peak value can be written as

$$
\left(k_{c 0}+m \Delta k_{0}\right) \frac{d}{\mathrm{Y}_{\mathrm{c}}} x=\frac{2 \pi}{N} h
$$

The image pixels in azimuth is still not uniform along range sample $m$, thus, a resampling in azimuth is needed so that

$$
k_{c 0} \frac{d}{\mathrm{Y}_{\mathrm{c}}} x=\frac{2 \pi}{N} h^{\prime}
$$

$h^{\prime}$ is the resampled image pixel in azimuth. Combine (9) and (10), one re-expresses the azimuth resampling in post azimuth Fourier transformed data domain as

$$
\frac{k_{c 0}}{k_{c 0}+m \Delta k_{0}} h=h^{\prime}
$$

In comparison of (8) and (13), $n$ and $n^{\prime}$ are the sample indices in wavenumber domain before azimuth FFT and $h$ and $h^{\prime}$ are the ones in image pixel after azimuth FFT. $n$ and $h$ are the sample indices before the resampling and $n^{\prime}$ and $h^{\prime}$ are the ones after the resampling. Also, since the azimuth FFT as (10) is applied prior to the azimuth resampling as (13), the entire azimuth samples that full-fill the data domain are utilized for the FFT. Thus, the zero padding is no longer needed, and the degradation of focusing quality from increase of sidelobes is avoided. Besides, to guarantee the modified PFA efficient, we adopt the scaling scheme to resample the data in the post Fourier transformed domain with the FFT based implementation [9]. After that, the final SAR image is obtained by applying IFFT in range. Fig. 1 illustrates the flowchart of the scaling scheme in FFT realization, where the coefficient

$$
\begin{array}{r}
\eta(m)=\frac{k_{c 0}}{k_{c 0}+m \Delta k_{0}} . \\
\quad \exp \left(-\mathrm{j} \pi \eta(m) \frac{h^{2}}{M}\right) \underset{\uparrow}{\longrightarrow} \rightarrow \text { IFFT } \\
\exp \left(j \pi \eta(m) \frac{h^{2}}{M}\right)
\end{array}
$$

Fig. 1. Flowchart of the scaling scheme based on FFT implementation. $(\otimes$ denotes complex multiplication)

\section{RESULTS}

Using the modified PFA discussed above, we simulated five scatterers aligned in a square, one at each corner and one in the center. The square is arranged in size of $260 \times 260 \mathrm{~m}$. In the simulation, we designed the SAR system with the transmitting parameter varying as the requirement in (6) with respect to the nominal center frequency of $9.6 \mathrm{GHz}$ and chirp rate of $5 \times 10^{13}$ $\mathrm{Hz} / \mathrm{s}$. The radar works on broadside spotlight mode in X-band, and the nominal bandwidth is $600 \mathrm{MHz}$, the pulse repetition frequency is $1250 \mathrm{~Hz}$, the speed of the platform is $105 \mathrm{~m} / \mathrm{s}$, the nearest distance from the SAR sensor to the center of an image scene is $15 \mathrm{~km}$, and the desired resolution is $0.3 \mathrm{~m} \times 0.3 \mathrm{~m}$.

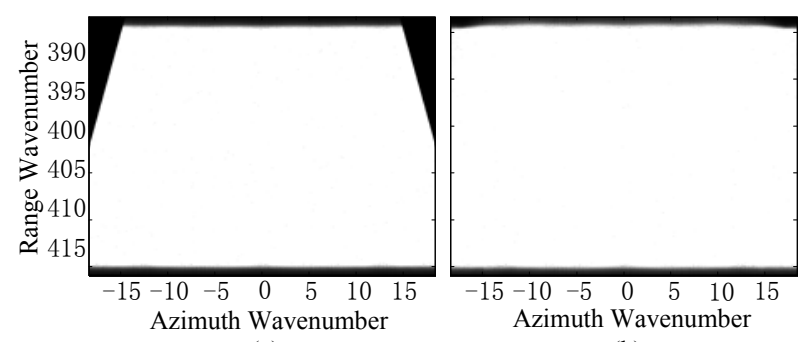

(a)

(b)

Fig. 2. Resampled data domain by conventional (a) and modified (b) PFA. 


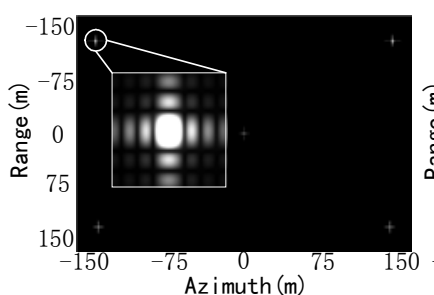

(a)

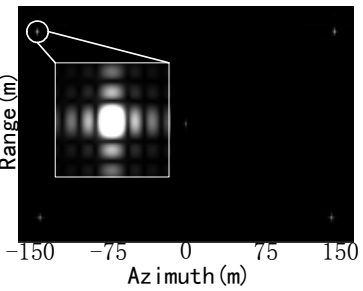

(b)
Fig. 3. SAR image by conventional (a) and modified (b) PFA.

According to the parameters listed above, the image size should be limited in a circle with $600 \mathrm{~m}$ diameter [7] by PFA image formation. Thus, the arranged square is sufficiently small than the limitation.

Fig. 2(a)-(b) are the resampled data in the 2-D wavenumber domain with the azimuth resampling in the pre- and postazimuth domains of the FFT, respectively. The data domain is unfilled in azimuth in Fig. 2(a), or zero-padded areas exist due to conventional PFA. Using the modified PFA, the data domain is completed full-filled in azimuth in Fig. 2(b). Fig. 3(a)-(b) show the SAR image processed by the conventional and modified PFA, respectively. To assess the performance of the developed PFA, we extract the scatterer as illustrated in Fig. 3 to generate its response in azimuth. The response shown as the (red) solid line in Fig. 4 is derived using the modified PFA, and the response indicated as a (black) dashed line using the conventional PFA. Quantitatively, the peak-sidelobe ratio (PSLR) is almost the same. The integrated-sidelobe ratio (ISLR) is better when the azimuth resampling is performed after the azimuth FFT than before the FFT (Fig. 4). The azimuth resolution corresponding to $3 \mathrm{~dB}$ width of the mainlobe in Fig. 4 is $0.2575 \mathrm{~m}$ which can meet the requirement of $0.3 \mathrm{~m}$. Also, in the post FT domain, the processing time for azimuth resampling is $13.31 \mathrm{~s}$ when the scaling is used but the time is $96.40 \mathrm{~s}$ if the interpolation employed, where the simulated data is in size of $2048 \times 2048$ samples.

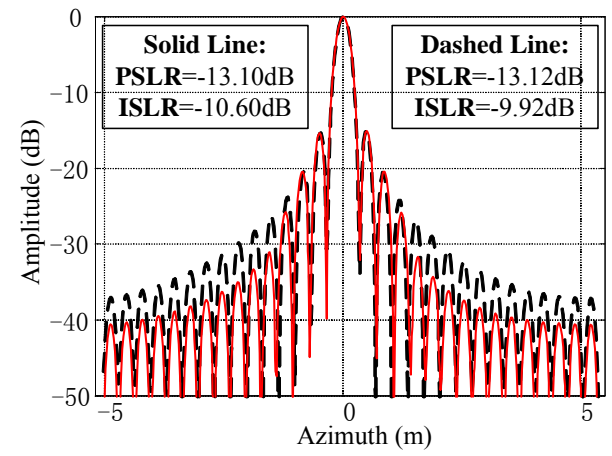

Fig. 4. Azimuth response by conventional (dashed line) and modified (solid line) PFA.

Finally, we process real spotlight SAR data by the proposed modified PFA. The data was downloaded from the Sandia Laboratory, and generated with the same parameters as those used in the simulation. A well-focused image of $400 \mathrm{~m} \times 400 \mathrm{~m}$ (rangexazimuth) is obtained (Fig. 5).

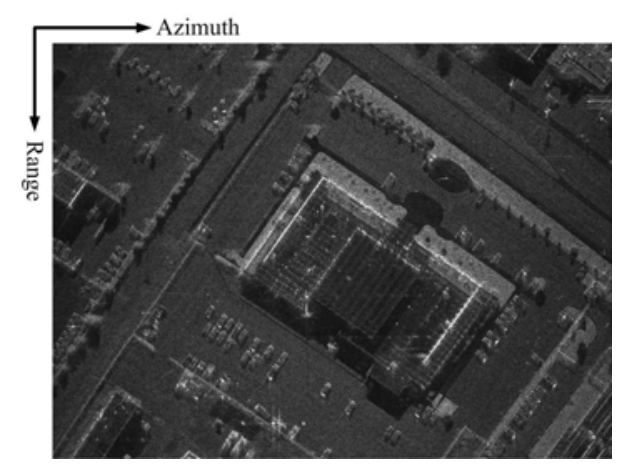

Fig. 5. SAR image with real spotlight data (processed spatial resolution: 0.3 $\mathrm{m} \times 0.3 \mathrm{~m})$

\section{CONCLUSION}

A modified PFA for the SAR image formation in spotlight mode with the azimuth resampling in the post Fourier transformed data domain is studied. Zero-padding in the azimuth resampled data domain is avoided, and focusing performance can be improved. The transmitting parameters variation is adopted to avoid range resampling, and scaling scheme in FFT implementation is utilized for achieving azimuth resampling in efficient. The algorithm is validated using simulated and acquired spotlight SAR data.

\section{ACKNOWLEDGMENT}

The authors thank the anonymous reviewers for their valuable comments to improve the paper quality, and the downloaded data from Sandia Lab. in supporting our study.

\section{REFERENCES}

[1] W. G. Carrara, R. S. Goodman, and R. M. Majewski. Spotlight Synthetic Aperture Radar: signal processing algorithms. Artech House, Norwood, 1995.

[2] R. Lanari, M. Tesauro, E. Sansosti, and G. Fornaro. Spotlight SAR data focusing based on a two-step processing approach. IEEE Trans. on Geosci. Remote Sens., vol. 39, no. 9, pp. 1993-2004, Sep. 2001.

[3] Y. Tang, M. D. Xing, and Z. Bao. The polar format imaging algorithm based on double Chirp-Z transforms. IEEE Geosci. Remote Sens. Lett., vol. 5, no. 4, pp. 610-614, Oct. 2008.

[4] A. W. Doerry. Patch diameter limits for tiered subaperture SAR image formation algorithms. In Proc. SPIE, vol. 2487, pp. 132-143, Jun. 1995.

[5] D. Zhu, S. Ye, and Z. Zhu. Polar format algorithm using chirp scaling for spotlight SAR image formation. IEEE Trans. on Aerosp. Electron. Syst., vol. 44, no. 4, pp. 1433-1448, Oct. 2008.

[6] T. A. Kennedy. Strapdown inertial measurement units for motion compensation for synthetic radar. IEEE Aerosp. Electron. Syst. Magazine, 1998, 3, (10), pp. 32-35.

[7] M. Preiss, D. Gray, and N. J. S. Stacy. Space variant filtering of polar format spotlight SAR images for wavefront curvature correction and interferometric processing. In Proc. IEEE Int. Geosci. Remote Sens. Symp., 2002, vol. 1, pp. 179-181.

[8] D. E. Wahl, P. H. Eichel, D. C. Ghiglia, and C. V. Jakowatz Jr. Phase gradient autofocus - a robust tool for high resolution phase correction. IEEE Trans. on Aerosp. Electron. Syst., vol. 30, no. 3, pp. 827-835, Jul. 1994.

[9] X. Lv, M. Xing, C. Wan, and S. Zhang. ISAR imaging of maneuvering targets based on the range centroid doppler technique. IEEE Trans. on Image Process., vol. 19, no. 1, pp. 141-153, Jan. 2010. 